\title{
“On-Chip” NanoFabricated Collagen Membranes Observed by High-Voltage Electron Microscopy
}

\author{
J.N. Turner*, D.H. Szarowski*, W. Shain", K. Buttle*, W.F. Tivol*, H. Bagle ${ }^{* *}$, A.J. Spence ${ }^{* *}$, S. \\ Retterer $^{* *}$, L. Lapek $^{* *}$, T. Richards**, M. Isaacson ${ }^{* *}$, M. Spencer ${ }^{* *}$ \\ * Wadsworth Center, New York State Department of Health, Albany, NY 12201-0509 \\ ** Cornell University, Ithaca, NY 14850
}

The field of NanoBioTechnology seeks to integrate biology with the technology of NanoFabrication. A wide variety of "on-chip" devices integrated with the associated biology are being developed. We can fabricate devices on the size scale of biological systems or molecules. These devices are fabricated using the highly parallel methods of nanofabrication. We are developing a collagen membrane system to perform filtration, and are integrating them into nanofabricated devices to perform "on-chip" separations. We have imaged the membranes in the high-voltage electron microscope (HVEM) using its acceleration potential to penetrate the specimen, and its physical size to mount centimeter scale chips.

The chip is fabricated from a silicon wafer (500 $\mu \mathrm{m}$ thick) on which $200 \mathrm{~nm}$ layers of $\mathrm{SiN}_{4}$ have been grown on both sides (Fig. 1). Squares 2, 4, $6 \& 8 \mu \mathrm{m}$ on a side are etched into the top layer by photo-lithography and a plasma etch, $1.5 \mathrm{~mm}$ holes in the bottom layer are fabricated in the same manner using a contact aligner with double sided alignment capability. This pattern serves as wet chemical etch stop to form pits in the wafer releasing the top $\mathrm{SiN}_{4}$ layer and the holes. Monomeric collagen (USBiological type I) was spun across the wafer 1 to 4 times and was fixed in $2 \%$ gluteraldehyde. Collagen membranes were stained in $2 \%$ Uranyl Acetate for imaging. The HVEM specimen rod tip (Fig. 2) was modified to have a large cut-out and a shelf against which the chip was clamped. This geometry allows a $2.5 \mathrm{~mm}$ wide region to be observed. If a $1 \mathrm{x} 1 \mathrm{~cm}$ chip is used it can be rotated $90^{\circ}$ to observe a second region. Membrane coated chips are shown in the insert of Fig. 2. One is mounted in the tip and the other is beside it. The rod can be rotated about it long axis to record different image perspectives. Parallax measurements determined that membranes with four spun layers were $400 \mathrm{~nm}$ thick. Fig. 3 shows low and high magnification images of collagen spun across a hole in the $\mathrm{SiN}_{4}$. Although the majority of this image is of the collagen across the through hole, structures at the edge were imaged through the $\mathrm{SiN}_{4}$. Pores from 25-100 nm were observed in the suspended collagen membrane. Some pores, black in the image, traversed the entire $400 \mathrm{~nm}$ thickness while those of higher intensity (brighter in the image) formed only through a portion of the thickness. Thus, it appears that thin collagen layers are spun across pores formed in earlier spins. We are currently studying the diffusion barrier characteristics of these membranes, their biocompatibility, and their ability to support selected cell growth.

This work was supported by the Nanobiotechnology Center, an STC of NSF (ECS-9876771) with assistance from the Wadsworth Center's Electron Microscopy Core. A.J. Spence: W.M. Keck Foundation Nanobiotechnology Program; S. Retterer: NSF Graduate Fellow. 


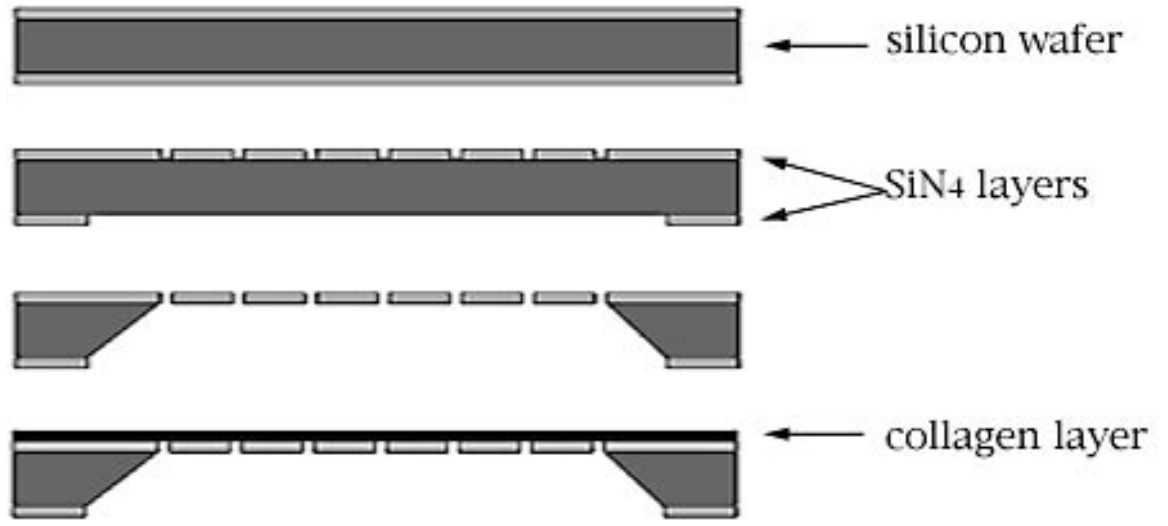

Fig. 1. A portion of silicon wafer showing the fabrication steps from top to bottom. Silicon wafer with $\mathrm{SiN}_{4}$ layers; fabrication of micrometer size holes by photolithography; release of holes in $\mathrm{SiN}_{4}$ layers by photolithography; collagen membrane spun on top of $\mathrm{SiN}_{4}$.

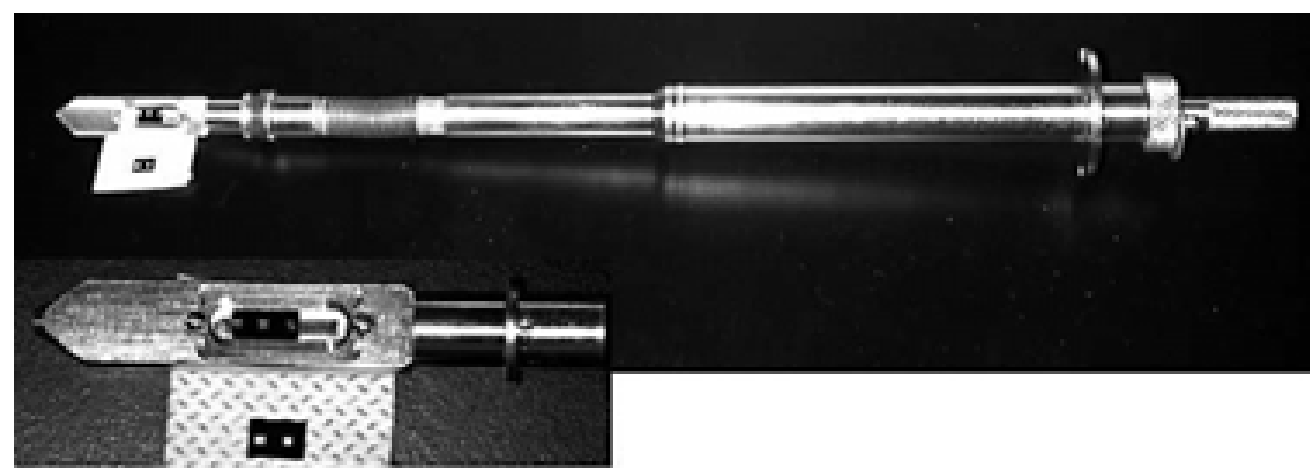

Fig. 2. HVEM specimen rod. Insert shows higher magnification view of chip holding tip and two chips with etched holes, one is mounted in the tip.
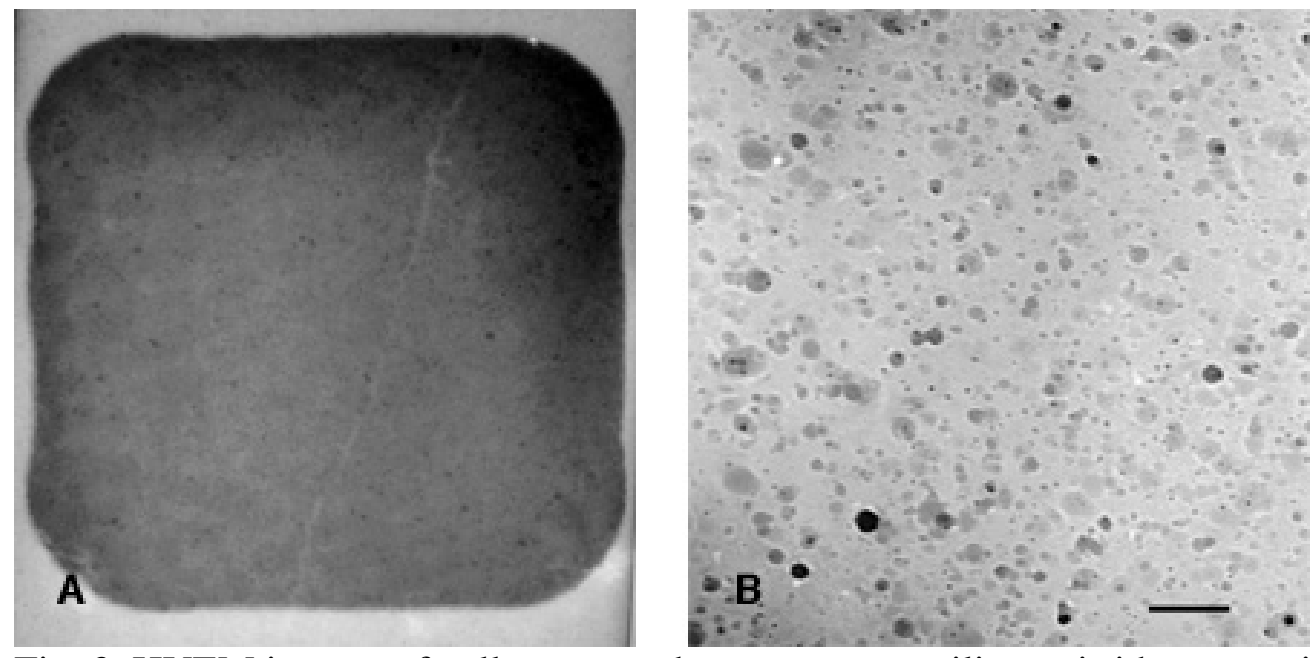

Fig. 3. HVEM images of collagen membranes spun on silicon nitride supporting membranes. A) is a low magnification of the through holes in the silicon nitride. B) is the pore structure of the collagen membrane spun across the silicon nitride through holes. The pores are $100 \mathrm{~nm}$ and smaller. These sizes are consistent with diffusion measurements on similar collagen membranes. Bar equals 200nm. 